\title{
Smooth Weighted Approach for Colour Histogram Construction using Human Colour Perception for CBIR Applications
}

\author{
Shaila S.G and A.Vadivel \\ Multimedia Information Retrieval Group \\ Department of Computer Applications, National Institute of Technology, \\ Tiruchirappalli 620 015, India, Fax: +91-431-2500133 \\ vadienitt.edu shailaenitt.edu
}

\begin{abstract}
In this paper, we construct histogram based on Human colour visual perception for Content-Based Image Retrieval. For each pixel, the true colour and grey colour proportion is calculated using a suitable weight function. During histogram construction, the hue and intensity value is iteratively distributed to the neighbouring bins. The NBS distance between the colour value of reference bin to the adjacent bins are estimated. The NBS distance value provides the proportion of overlap of colour of the reference bin with the adjacent bins and accordingly the weight is updated. This kind of procedure for constructing the histogram uses minute colour information and captures the complex background colour content. The distribution makes it possible to extract the background colour information effectively along with the foreground information. The low-level feature of all the database images are extracted and stored in feature database. The relevant images are retrieved for a query image based on the similarity ranking between the query and database images and Manhattan distance is used as similarity measure. The performance of the proposed approach using coral benchmark dataset is encouraging and the precision of retrieval is compared with some of the similar work.
\end{abstract}

\section{Keywords}

Human colour perception, Smooth distribution, NBS distance, background complex.

\section{Introduction}

Various techniques have been proposed in academia and industry for image retrieval applications. These approaches can roughly be classified into three categories such as text-based retrieval, content-based retrieval and semantic-based retrieval. In the text-based retrieval technique, each image has a number of keywords for describing the image and the keyword based matching is performed to retrieve relevant images. In content-based image retrieval applications, various wellknown low-level features like colour, texture and shape are extracted for describing the image semantics. In the semantic-based retrieval technique, semantics are used to retrieve the relevant images. In recent years, attention is focused by researchers on Content-Based Image Retrieval (CBIR), which is a sub-problem of content-based retrieval. It is noticed that the size of the image database used in image retrieval is increasing exponentially and hence it is necessary to propose and use effective tools for retrieving relevant images. Well-known and most popular image retrieval systems are QBIC[13], NeTra[11], PicToSeek[6], Blobworld[1], etc. Human visual system plays a vital role in the colour theory of image retrieval applications as human eyes first captures colour. There are two types of cells in human retina, namely the rod cells and the cone cells and are responsible for the sensitivity of the light. Rod 
The International Journal of Multimedia \& Its Applications (IJMA) Vol.4, No.1, February 2012

Cells are responsible for grey vision and cone cells are responsible for colour vision. Colour vision results from the action of three cone cells with different spectral sensitivities at Red (R), Green $(\mathrm{G})$ and Blue (B) of the visible light spectrum. The peak sensitivities of these three cone cells are located approximately at $610 \mathrm{~nm}, 560 \mathrm{~nm}$ and $430 \mathrm{~nm}$, respectively. When particular wavelength of a light is incident on the eye, the cells are stimulated to different degrees, and the ratio of the activity in the three cells results in the perception of a particular colour. Each colour is therefore coded in the nervous system by its own ratio of activity in the three types of cone cells. For a given wavelength of light, human colour perception is determined by which combination of cones is excited and by how much [21].

In this paper, our goal is to retrieve set of images that are similar to a given query image. In image retrieval applications, it has been observed that the colour histogram based approach is well suited, since colour matching generates the strongest perception of similarity to the human eye. It is often represented in the form of a histogram, which is a first-order statistical measure that captures the global distribution of colour in a given image. This can be represented in various forms such as colour histogram [15], colour moments and cumulative colour histogram [16]. Cumulative colour histogram use the spatial relationship between histogram bins . A colour histogram may be generated in the RGB colour space, HSV colour space, YCbCr colour space, etc. Since, the RGB colour space is having some drawbacks such as, it does not explicitly distinguish between colour and intensity components, other colour space like HSV, YCbCr, which separate saturation and intensity components are frequently used. RGB values by suitable conversions may be computed to get the values for each component in HSV colour space [20]. It is also possible to generate three separate histograms, one for each channel, and concatenate them into one [8].Some of the recently proposed colour representatives are colour saliency histogram [23], which supports human visual attention principle and based on the features of colour, orientation and intensity and followed by the difference of Gaussians and normalization processing, the comprehensive saliency map of an image is generated. Recently, circular ring histogram [22] has been proposed, which has the spatial information. The image is segmented initially using group of circular rings and then the histogram is constructed using the segmented rings. The statistical feature of image blocks has been extracted for representing the colour of blocks, which is named as Order Based Block Colour Feature [19], in this approach image is divided into 48 blocks and the feature is extracted. Since histogram components store the number of pixels having similar colours, it may be considered to be a signature of the complete image represented by a feature vector. In our proposed work, we propose new colour histogram construction scheme based on human colour perception and NBS distance to capture the colour information in high-dense background of images for retrieval applications. Pixel weight is distributed to the neighbouring bins based on NBS distance and the performance of the proposed approach is encouraging while retrieving high-dense background images. During retrieval, histogram of query image is compared with histograms in feature database. The histogram is a point in the n-dimensional vector space and a distance measure like the Manhattan distance is used for the comparison and ordering of these vectors.

The outline of this paper is as follows. Section 2 presents the related works and proposed technique is explained in Section 3. In Section 4, the experimental results are presented and we conclude the paper in the last section.

\section{Related Works}

In colour-based image retrieval, there are primarily two methods: one based on colour layout [18] and the other based on colour histogram [3]. In the colour layout approach, two images are matched by their exact colour distribution. This means that two images are considered close if they not only have similar colour content, but also if they have similar colour in approximately the same positions. In the second approach, each image is represented by its colour histogram. A 
histogram is a vector, whose components represent a count of the number of pixels having similar colours in the image. Thus, a colour represents to be a signature extracted from a complete image. Colour histograms extracted from different images are indexed and stored in a database. During retrieval, the histogram of a query image is compared with the histogram of each database image using a standard distance metric like Euclidean distance or Manhattan distance [20]. Since, colour histogram is a global feature of an image, the approaches based on colour histogram are invariant to translation, rotation and scale.

Various techniques has been proposed to represent the colour of an image [2][7][9][10][14][17]. Gevers and Stokman [5] have proposed a histogram for object recognition problem. A variable kernel density estimation is used in this approach to construct colour invariant histograms. Uncertainty associated with colour variant values of hue and normalised RGB colour are computed and used to determine the kernel sizes. In Pyramid Histogram Of TOpics (PHOTO) [4] the image is represented to suit the classification requirement and image is partitioned into hierarchical cells. The topic histogram is learned using pLSA with EM algorithm. The topic histograms are concatenated over the cells at all levels to form a long vector, i.e. pyramid histogram of topics. In fuzzy colour histogram [7] is constructed by considering the colour similarity of each pixel's colour associated to all the histogram bins through fuzzy-set membership function. A fast approach for computing the membership values based on fuzzymeans algorithm is introduced to identify objects based on the colour histograms. Swain and Ballard [15] propose a histogram intersection method, which is able to eliminate the influence of colour contributed from the background pixels during the matching process in most cases. Although their method is robust to object occlusion and image resolution, it is still sensitive to illumination changes. It is found that robust histogram construction scheme using the HSV colour space in which a perceptually smooth transition is captured based on the Human Visual Perception of colour $(\mathrm{HCPH})$ can represent semantic information up to a certain degree, due to the complex background [21]. In the DCT Histogram Quantization [12], the method extracts and constructs a feature vector of histogram quantization from partial DCT coefficient in order to count the number of coefficients that have the same DCT coefficient over all image blocks. The database image and query image is equally divided into a non overlapping $8 \mathrm{X} 8$ block pixel, each of which is associated with a feature vector of histogram quantization derived directly from discrete cosine transform DCT which results good results for clear background.In colour saliency histogram [24], extraction of salient regions is based on the bottom-up visual attention model. Although it has introduced prior knowledge in the model, the bottom-up attention is only suitable for the primary stage of visual perception, it has limitations. Order-based Block Colour Feature [19] is one type of image's colour feature. It has an advantage of the local colour statistical information but it has some bionic traits that this colour feature cannot alone suffice for CBIR. It has to be combined with other features such as shape, texture, and so on.

Based on the above discussion, it is noticed that each method used various techniques and none of them uses the human visual perception. However though HCPH uses human visual perception, it fails to discriminate the background colour with the foreground colour for better retrieval. Thus, in the proposed approach we combine both human visual perception and NBS distance for iteratively distribute the colour to the adjacent bins. It is noticed that this procedure of updating histogram bins effectively discriminates the foreground with the background.

\section{Proposed Approach}

The HSV colour model is one of the colour models that separate out the luminance component, intensity of a pixel from its chrominance components Hue and Saturation. This representation is more similar to the human perception of colour through rod and cone cells. Hue represents pure colours, and are perceived by the excitation of cone cells when incident light is of sufficient 
illumination as well as contains a single wavelength. Saturation gives a measure of the degree by which a pure colour is diluted by white light. Light containing such multiple wavelengths causes different excitation levels of the cone cells resulting in a loss of colour information. For light with low illumination, corresponding intensity value in the HSV colour space is also low. Only the rod cells contribute to visual perception at low intensity illumination with little contribution from the cone cells.Pixels in a image can be represented with the combination of Hue, Saturation and Intensity Value in the HSV colour space. It is a three dimensional hexacone representation and the central vertical axis is intensity, I. Hue is an angle in the range $[0,2 \pi]$ and is relative to the red axis with red at angle 0 , green at $2 \pi / 3$, blue at $4 \pi / 3$ and red again at $2 \pi[23]$ respectively. Saturation, $S$, is measured as a radial distance from the central axis with value between 0 at the centre to 1 at the outer surface [21]. This is represented in Fig.1.

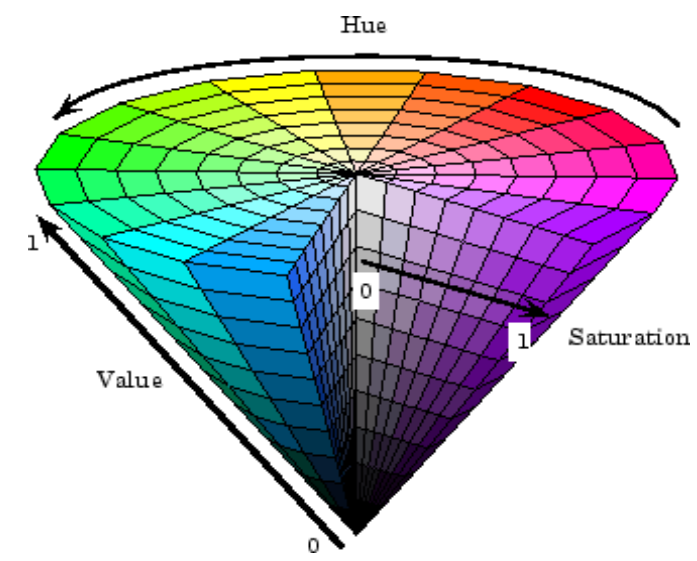

Fig 1. HSV Colour Model

The pixel colour is approximated by its intensity or by its hue, say for example while the intensity is low and saturation is high, a pixel colour is very much close to the grey colour. Similarly for other combination of intensity and saturation, we can approximate the pixel value the other way. Therefore, the saturation and intensity values of a pixel are used to determine whether it can be treated as a true colour pixel or a gray colour pixel. It is emphasized that this approach treats the pixels as a distribution of "colours" in an image where a pixel may be of a "gray colour" (i.e., somewhere between black and white, both inclusive) or of a "true colour" (i.e., somewhere in the red, green, blue, red spectrum). The reason is that, for an observer, this is what an image represents is a collection of points having colours - red, yellow, green, blue, black, gray, white, etc.

In our proposed work, colour information of each pixel is converted to HSV colour space. An image pixel contains true colour components, in which the dominant factor is hue. Intensity is the dominant factor for grey colour components. In the first step, we separate the pixel colour information into true colour components and grey colour components using a weight function[21] given in Eq.(1) and Eq.(2)

$$
\begin{aligned}
W_{H}(S, I) & =\left\{\begin{array}{lll}
S^{r 1(255 / I)^{r 2}} & \text { for } & I \neq 0 \\
0 & \text { for } & I=0
\end{array}\right. \\
W_{I}(S, I) & =1-W_{H}(S, I)
\end{aligned}
$$




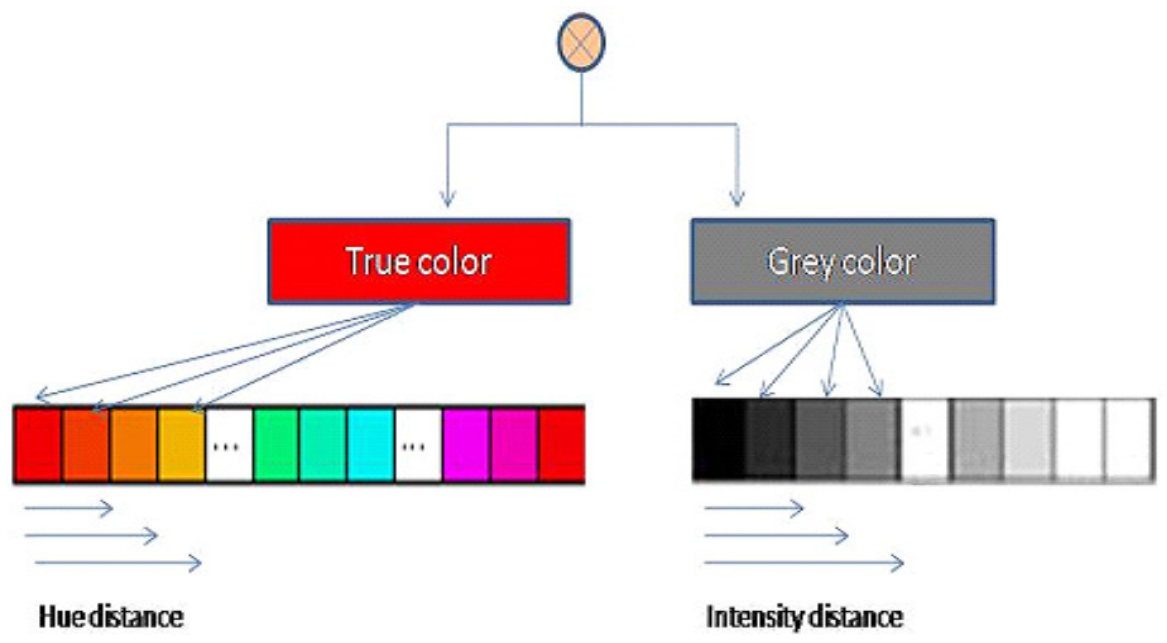

Fig 2. Distribution of true colour and grey colour components

As noticed from Fig. 2, the assumption is the true colour component is updated in the first bin of the true colour part and the grey colour component is updated in the first bin of the gray colour part. The distance between the reference bin and its smoothly distributed corresponding adjacent bins is computed by applying NBS distance formula [23] represented by Eq. (3). For true colour, $\Delta \mathrm{S}$ and $\Delta \mathrm{I}$ remains zero. This is due to the fact that the saturation and intensity distribution difference is invariable or minimal with respect to a single pixel. For grey colour, $\Delta \mathrm{S}$ and $\Delta \mathrm{H}$ remains zero, since saturation and hue difference appears to be very minimal or invariable.

$$
d(\vec{x}, \vec{y})=1.2 * \sqrt{2 x_{2} y_{2}\left(1-\cos \left(\frac{2 \pi \Delta H}{100}\right)\right)+\Delta S^{2}+(4 \Delta I)^{2}}
$$

Table.1. NBS distance Table

\begin{tabular}{|l|l|}
\hline NBS Value & Human Perception \\
\hline $0 \sim 1.5$ & Almost the same \\
\hline $1.5 \sim 3.0$ & Slightly different \\
\hline $3.0 \sim 6.0$ & Remarkably different \\
\hline $6.0 \sim 12.0$ & Very different \\
\hline $12.0 \sim$ & Different colour \\
\hline
\end{tabular}

Based on the ranges, weights are assigned to true colour components and grey colour components. It is observed that the immediate adjacent bin close to the reference bin will lie in the distance ranging between $0 \sim 1.5$, which represents the colour difference as "almost the same" as per the NBS table. Thus, $100 \%$ of true colour weight held by reference bin will be distributed to the immediate adjacent bin. As the distance of the adjacent bins increase, the colour difference will also increase proportionately. If the distance lies in the range of 1.6 3.0, we observe that a "slight colour difference", and we divide it into 3 groups with 15 iterations. Now, the weight is 
The International Journal of Multimedia \& Its Applications (IJMA) Vol.4, No.1, February 2012

distributed in the range between $99 \%$ to $85 \%$ of reference true colour weight. When the distance is measured with respect to the adjacent bins in the range of 3.1 6.0, there is a "remarkable colour difference" range. Hence, we have divided it into 6 groups with 30 iterations and weight distribution starts in the range of $84 \%$ to $55 \%$ of reference true colour weight. As distance increases, the colour difference will be more, which may lie in the range of 6.1 12.0 and is represented as "very different colour". We divide it into 12 groups with 60 iterations and weight distribution starts in the range of $54 \%$ to $0 \%$, if distance is greater than 12.0, then it represents totally different colour and there will be no weight distribution or $0 \%$ weight distribution and is represented in the form of tree in Fig.4.

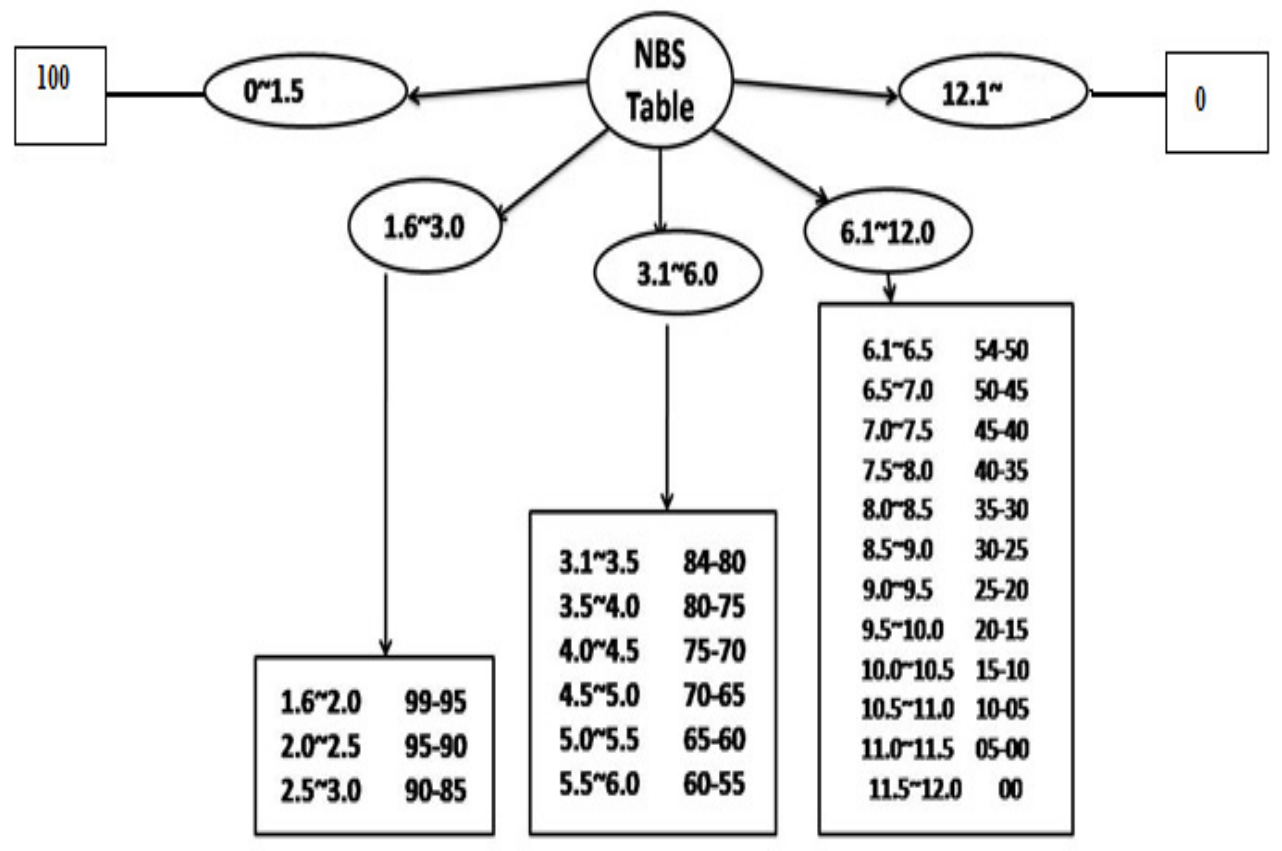

Fig 4. Construction of Smooth Weight Distribution Tree

The weight distribution percentage for the true colour to the adjacent bins is calculated using Eq. (4)

$$
S W_{H}(S, I)=100-[(N B S-1.5) * 10]
$$

During grey colour weight distribution, intensity is distributed smoothly. NBS distance is not applicable for grey colour weight distribution, since the colour difference is very minimum and is not rectified. But distribution of grey colour weight is done by using Eq. (5).

$$
S W_{I}(S, I)=1-S W_{H}(S, I)
$$

$$
\begin{aligned}
& \text { Where NBS - True colour distance } \\
& S W_{H}(S, I) \text { - Smooth true colour weights } \\
& S W_{I}(S, I) \text { - Smooth grey colour weight }
\end{aligned}
$$


The International Journal of Multimedia \& Its Applications (IJMA) Vol.4, No.1, February 2012

ALGORITHM

For each pixel in the image,

Read RGB value

Convert RGB to HSV colour space

Determine the weight function for true colour $W_{H}(S, I)$ and grey colour $W_{I}(S, I)$ using Eq.(1) and Eq.(2)

Smoothly distribute true colours and grey colours among adjacent bins iteratively.

Determine the index of the bins for smoothly distributed true colour(Hue) and grey colour(Intensity) using MULT_FCTR and DIV_FCTR

Compute NBS distance between reference colour bin and its corresponding smoothly distributed adjacent bins using NBS distance formula

Depending on the computed distance of reference colour bin and its corresponding smoothly distributed adjacent bins Find the colour difference using NBS distance Table.

Determine the weight function for smoothly distributed true colour $S W_{H}(S, I)$ and weight function for smoothly distributed grey colour $S W_{I}(S, I)$ among the bins using Smooth Weight Distribution Tree

Update the histogram for true colour and grey colour as follows:

Smooth True Colour Hist [Round (Smoothly distributed Hue* MULT_FCTR)]=

Smooth True Colour Hist [Round (Smoothly distributed Hue* MULT_FCTR))]+

$S W_{H}(S, I)$

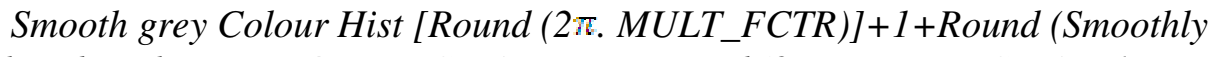
distributed Intensity/DIV_FCTR $)=$ Hist $[$ Round $(2 \pi$. MULT_FCTR $)]+1+$ Round (Smoothly distributed Intensity/DIV_FCTR) $+S W_{I}(S, I)$

Similarly, the distance between the first grey to the adjacent bins are also calculated. Using the distance value, the proportion by which the current pixel colour overlaps with neighbouring colour is estimated and accordingly the weight of the bins is updated. 


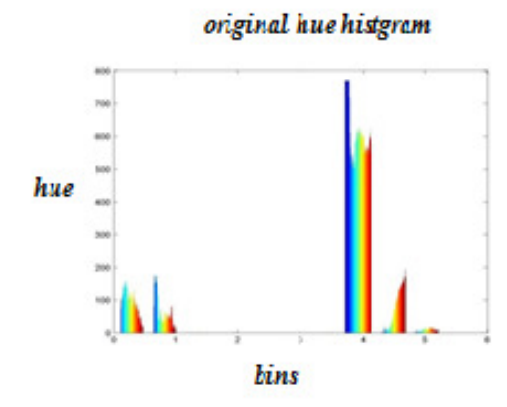

(a)

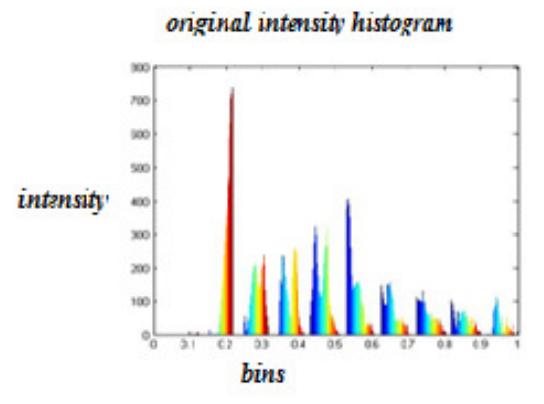

(c)

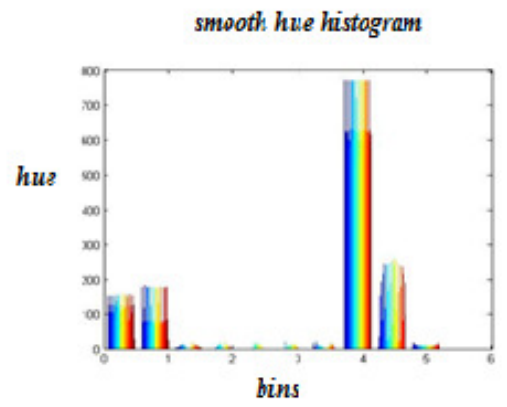

(b)

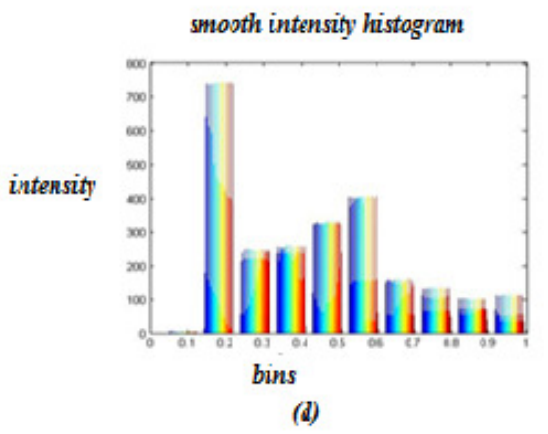

Fig.3. Smooth Distribution of hue and intensity

The histogram representation of HCPH shown in Fig.3(a) and 3(c). Here, pixel containing true colour information and grey colour information is grouped into two separate bins. The Graphical representation of smooth distribution of true colour and grey colour using proposed approach is shown in Fig.3(b) and 3(d) which represents soft nature of distribution. From the figure, it can be observed that the proposed approach has flat bit representation and thus has more information about the colour and intensity of image.

\section{Experimental Results}

The effectiveness of the proposed histogram is tested in an image retrieval system. The performance evaluation measures for determining the accuracy of such systems include recall and precision. It is difficult to have comparative analysis of CBIR schemes without the benchmark image databases. Without the ground truth, it is hard to calculate recall measures on such databases. In our experiments, we have taken a coral benchmark database of about 10,000 images of various classes such as people, vehicle, building, flower, etc.. We have selected set of query images having dense and complex background from these 10,000 images for computing recall and precision. Precision is the ratio of the number of the relevant images retrieved to the total number of images retrieved.

$$
\operatorname{Precision}(\mathrm{P})=\frac{R_{r}}{T}
$$

Where $R_{r}$ is number of relevant images retrieved. $T$ is the total images retrieved 
The International Journal of Multimedia \& Its Applications (IJMA) Vol.4, No.1, February 2012

Recall is defined as the ratio of the number of relevant images retrieved to the total number of the relevant images in the database. Recall is a measure of completeness.

$$
\operatorname{Recall}(\mathrm{R})=\frac{R_{\gamma}}{T_{r}}
$$

Where $R_{r}$ is number of relevant images retrieved.

$T_{r}$ is the total number of relevant images in the database

Here the comparison is done between $\mathrm{HCPH}$ and proposed method and the results are shown. We have considered top 50 images retrieval and the average precision and recall graph for 10 different classes are shown in Fig.5 and Fig.6. The results for dense background images are obtained progressively.

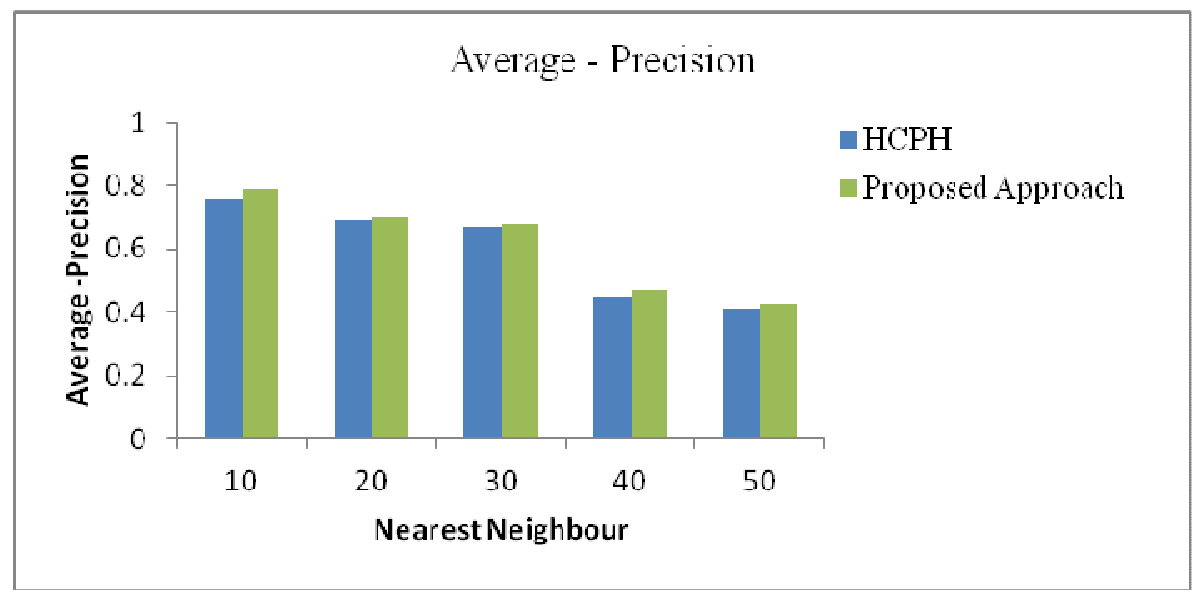

Fig.5 Average precision

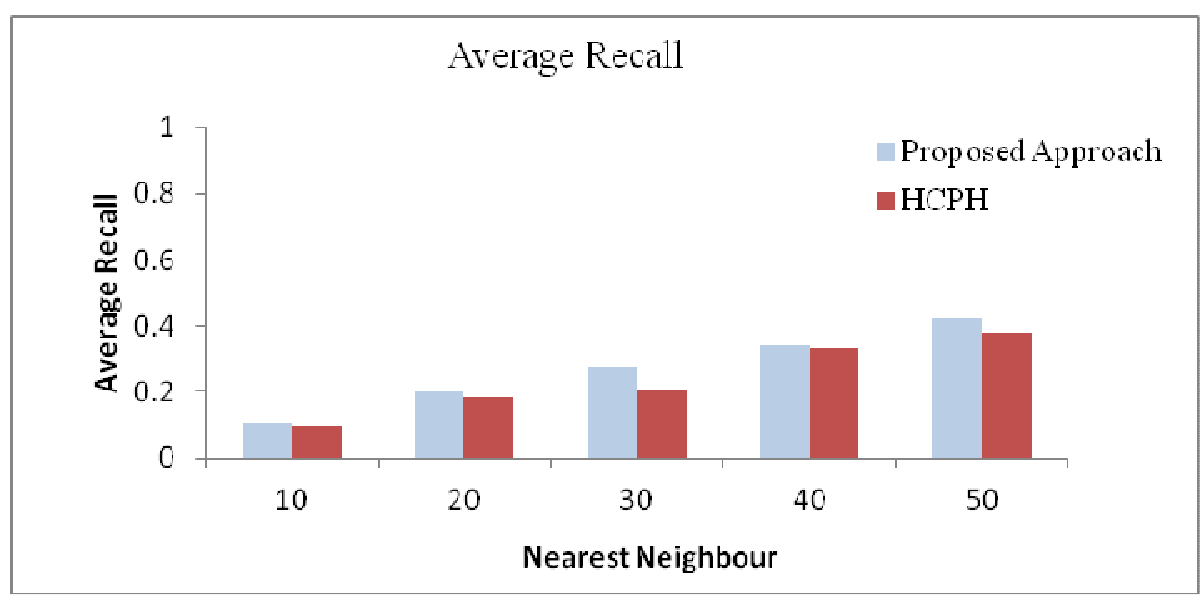

Fig.6 Average Recall 
The International Journal of Multimedia \& Its Applications (IJMA) Vol.4, No.1, February 2012

Average Precision vs. Recall is calculated and is shown in the Fig.7. Results are obtained for different values of recall from 0.1 to 1.0 in steps of 0.1 and the corresponding precision was calculated. For all the methods, Manhattan distance was used as the distance metric. The higher the distance, the lower is the similarity between a query image and a target image. From the figure, It is observed that for lower values of recall, the precision is getting higher, which is reaching $80 \%$. Similarly, for higher value of recall, the precision is comparable and the performance of the proposed method is encouraging.

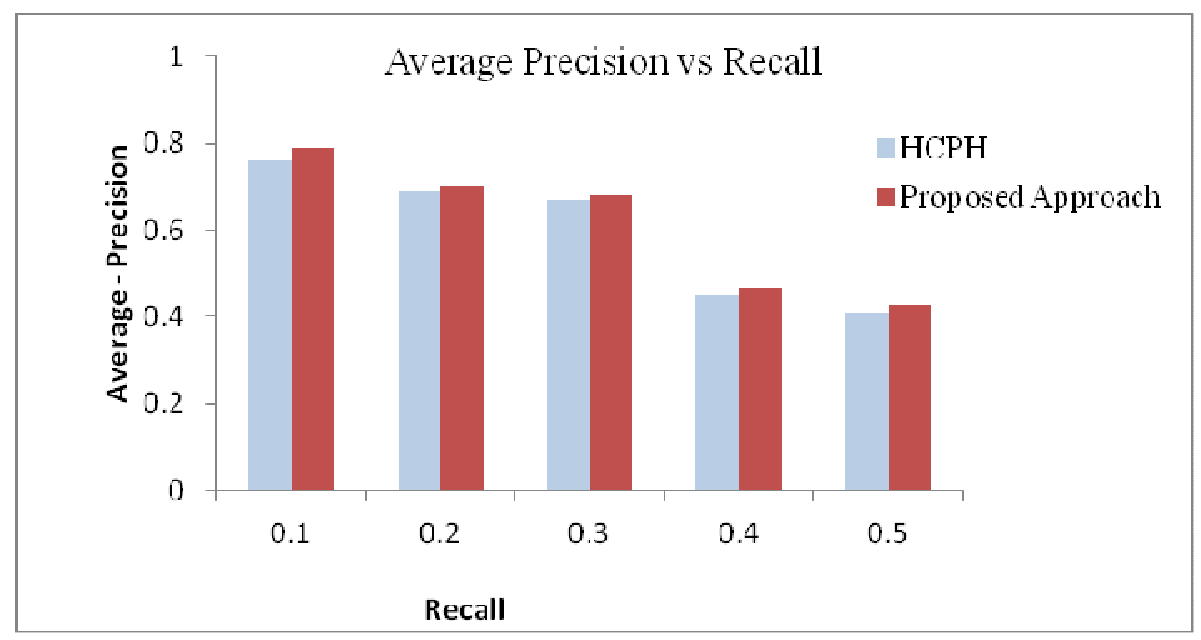

Fig 7. Average Precision versus Recall

Further we have F-Measure for considering both recall and precision into account and is given in Eq.(8).This is an Harmonic mean of recall and precision and is shown in Fig.8.

$$
F=\left(\frac{2 P R}{P+R}\right)=\left(\frac{2}{\frac{1}{R}+\frac{1}{P}}\right)
$$

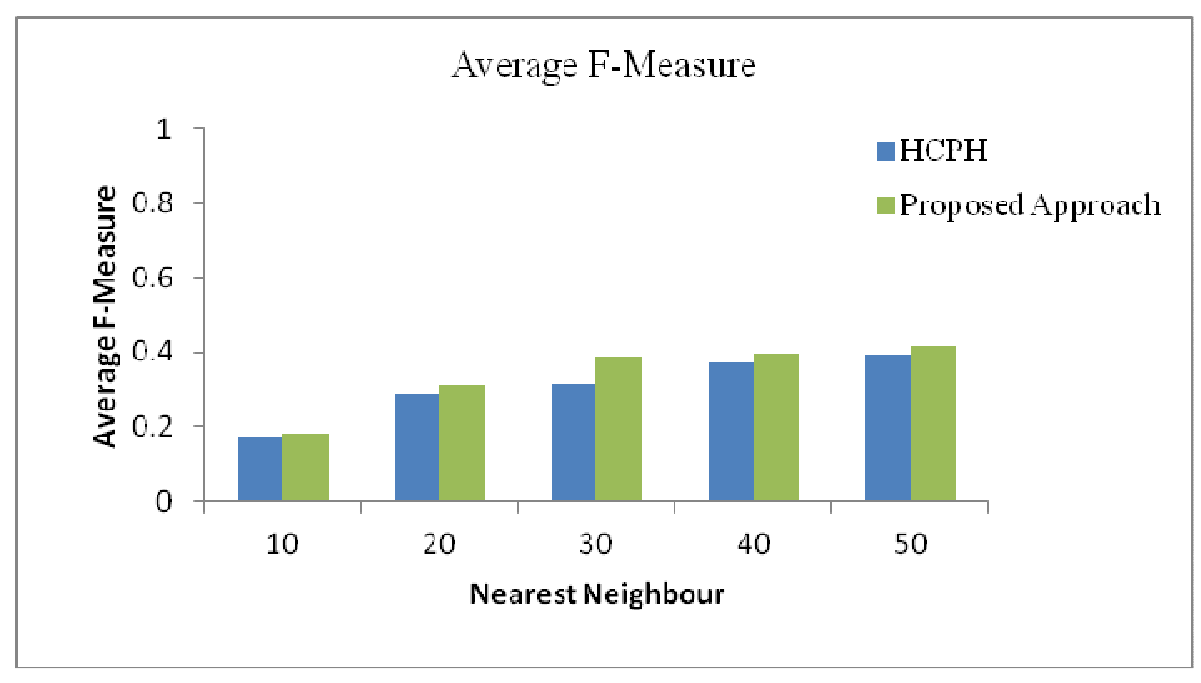

Fig.8. Average F-measure 
The International Journal of Multimedia \& Its Applications (IJMA) Vol.4, No.1, February 2012

Since, weight is smoothly distributed to neighbouring bins based on the true colour and gray colour weight, the entire information of the background as well as the object is captured and thus the precision retrieval is 80\%. Fig.9 and Fig.10 depicts sample retrieval result for both the proposed and HCPH histogram. The image on the top centre is the query image and the rest of the image below is the retrieved image set. In our proposed method background and foreground Colours are similar for query image in the top 4 retrieval set. But in HCPH this is appeared only in top 2 retrieval set.

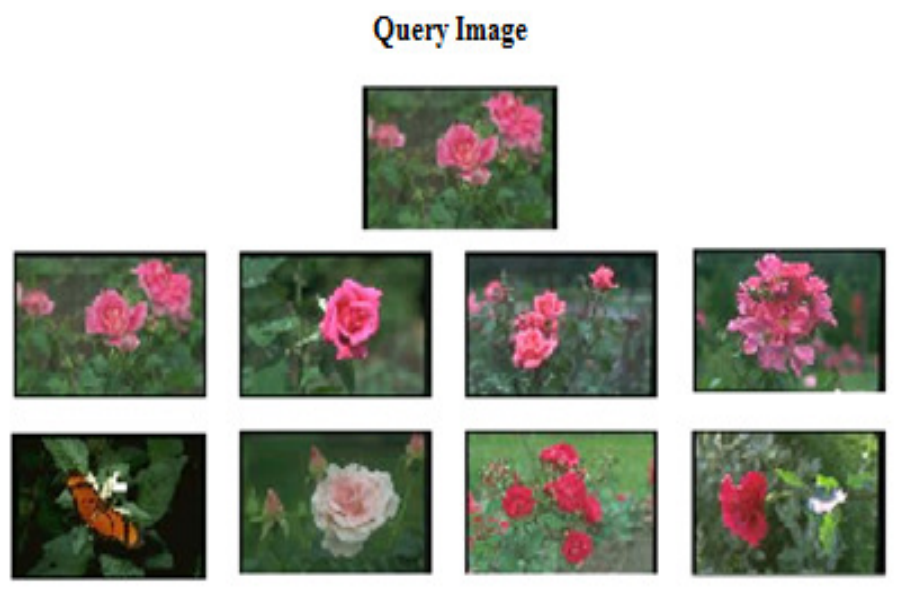

Fig 9. Sample Retrieval set using Proposed approach

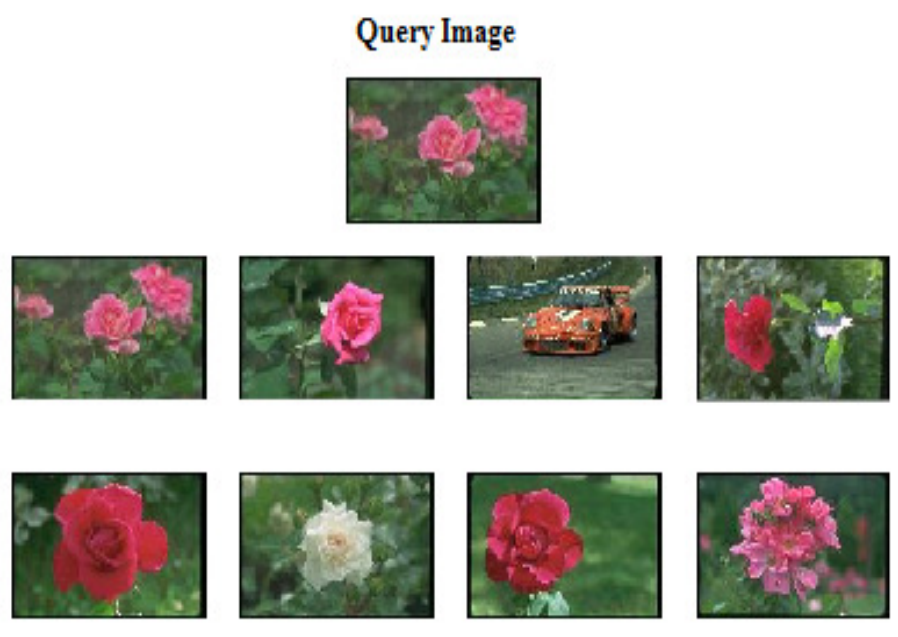

Fig 10. Sample Retrieval set using HCPH.

HCPH algorithm is compared with two methods proposed in Gevers and Stokman (2004) and colour-based co-occurrence matrix scheme (MCCM) proposed in Shim and Choi (2003).It is observed that the HCPH method outperforms by more than $15 \%$. When Proposed approach is compared with $\mathrm{HCPH}$ it is found that the Proposed approach algorithm outperforms between $3 \%$ to $5 \%$ for dense background images. It is noted that when tested with a large image database having wide variety of images, precision of all the image retrieval techniques deteriorates 
The International Journal of Multimedia \& Its Applications (IJMA) Vol.4, No.1, February 2012

considerably. This is balanced in our results when tested on 10,000 images, which have been categorised into different groups. A high percentage of these relevant images are retrieved for each query, resulting in high precision and recall. However, even with uncontrolled image database, performance of the proposed method is comparatively better than the other approaches.

\section{Conclusion}

In this paper, we have proposed new colour histogram construction technique .The colour features of each pixel of the image is smooth distributed to the neighbouring bins iteratively. This representation of colour distribution of an image captures the foreground and background information of an image effectively. This is due to the fact that this approach uses both human visual perception and NBS distance measure. The performance of the proposed approach is encouraging in benchmark datasets and it is found that the precision of retrieval is higher for background complex images. For future work, we plan to improve the rate of retrieval by adding more iterations and adding extra features such as texture and shape with the colour to improve the relevance by effectively balancing both of these parameters.

\section{Acknowledgment}

This work is supported by research grant from the Department of Science and Technology, India, under Grant DST/TSG/ICT/2009/27 dated 3rd September 2010.

\section{References}

[1] Carson, C., Thomas, M., Belongie, S., Hellerstein, JM, \& Malik, J, (1999) "Blobworld: A system for region-based image indexing and retrieval", Third Int. Conf. on Visual Information Systems, pp $217-$ 225 .

[2] Deng, Y., Manjunath, B.S., Kenney, C., Moore, M.S. \& Shin, H. (2001) "An efficient colour representation for image retrieval”, IEEE Transactions on Image Processing, Vol. 10, pp140-147.

[3] Deb \& Y. Zhang, (2004) "An overview of content-based Image retrieval techniques," Proc. on 18th Int. Conf. on Advanced Information Networking and Applications, Vol. 1, pp59-64.

[4] Fuxiang Lu, Xiaokang Yang, Rui Zhang, \& Songyu Yu , (2009) "Image classification based on pyramid histogram of topics,". IEEE International Conference on Multimedia and Expo, ICME 2009 , pp398-401.

[5] Gevers, T. and Stokman, H.M.G. (2004) 'Robust histogram construction from colour invariants for object recognition', IEEE Transactions on Pattern Analysis and Machine Intelligence, Vol. 26, pp.113-118.

[6] Gevers,T \& Smeulders, A.W.M., (2000) "PicToSeek: Combining colour and shape invariant features for image retrieval”, IEEE Trans. on Image Processing, Vol. 9 pp102-119.

[7] Han, J. \& Ma, K-K., (2002) "Fuzzy colour histogram and its use in colour image retrieval", IEEE Transactions on Image Processing, Vol. 2, pp944-952.

[8] Jain, A \& Vailaya,A ,(1996) " Image retrieval using colour and shape”, Pattern Recognition, Vol. 29 pp1233-1244.

[9] Kender, J.R., (1976) "Saturation, Hue and Normalised Colour: Calculation, Digitisation and Use", Computer Science Technical Report, Carnegie-Mellon University, Pittsburg, USA.

[10] Lei, Z., Fuzong, L. \& Bo, Z., (1999) “A CBIR method based colour-spatial feature”, Proc. IEEE Region 10 Annual International Conference on TENCON 99, Proceedings ,Cheju Island, South Korea, pp166-169.

[11] Ma W.Y. \& Manjunath, B.S., (1997) "NeTra: A toollative box for navigating large image databases", IEEE Int. Conf. on Image Processing pp568-571.

[12] Mohamed, A., Khellfi, F., Ying Weng, Jianmin Jiang \& Ipson, S., (2009) "An efficient Image Retrieval through DCT Histogram Quantization" , International Conference on CyberWorlds, pp237-240. 
The International Journal of Multimedia \& Its Applications (IJMA) Vol.4, No.1, February 2012

[13] Niblack,W, Barber,R Equitz,W, Flickner,M, Glasman,E,.Petkovic,D Yanker,P,.Faloutsos,C \& Taubin,G., (1993) "The QBIC project: Querying images by content using colour, texture and shape", SPIE Int. Soc. Opt. Eng., in Storage and Retrieval for Image and Video Databases.Vol. 1908, pp173187.

[14] Nezamabadi-pour, H. \& Kabir, E., (2004) "Image retrieval using histograms of uni-colour and bicolour blocks and directional changes in intensity gradient", Pattern Recognition Letters, Vol. 25, pp1547-1557.

[15] Swain, M.J \& Ballard, D.H., (1991) “Colour Indexing”, Computer Vision. Vol. 7, pp11-32.

[16] Stricker, M A. \& Orengo, M., (1995) “Similarity of colour images”, SPIE, Vol. 2420, pp381-392.

[17] Shih, J.L. \& Chen, L.H., (2002) "Colour image retrieval based on primitives of colour moments", Proc. IEEE Vision, Image and Signal Processing, pp88-94.

[18] Smith, J. R., \& Chang, S.-F., (1996) "VisualSEEk: A Fully Automated Content-Based Image Query System", ACM Multimedia, pp87-98

[19] Shoujue Wang \& Hong Qin , (2009) "A Study of Order-Based Block Colour Feature Image Retrieval Compared with Cumulative Colour Histogram Method", Sixth International Conference on Fuzzy Systems and Knowledge Discovery, Vol. 1, pp81-84.

[20] Vadivel A. ,Majumdar A. K. \& Sural Shamik, (2003) "Perceptually Smooth Histogram Generation from the HSV Colour Space for Content Based Image Retrieval", International Conference on Advances in Pattern Recognition, Kolkata, pp 248-251.

[21] Vadivel.A, Shamik Sural \& A.K.Majumdar, (2008) "Robust Histogram Generation from the HSV Space based on Visual Colour Perception", International Journal of Signal and Imaging Systems Engineering, InderScience, Vol. 1(3/4), pp245-254.

[22] Wang \& Xiaoling, (2009) "A Novel Circular Ring Histogram for Content-Based Image Retrieval", First International Workshop on Education Technology and Computer Science, Vol. 2, pp785-788.

[23] Yihong Gong, Proietti, G \&, Faloutsos, C., (1998) "Image indexing and retrieval based on human perceptual colour clustering,". Proc. IEEE Computer Society Conference on Computer Vision and Pattern Recognition, pp.578-583.

[24] Yunqi Lei, Zhenxiang Shi, Xutuan Jiang, Qingmin Li \& Dongjie Chen , (2009) "Image Retrieval Based on Colour Saliency Histogram", International Symposium on Computer Network and Multimedia Technology, pp1-4.

\section{Authors}

\section{Short Biography}

A. Vadivel is an Associate Professor at the National Institute of Technology, Tiruchirappalli, India. He received his MTech and $\mathrm{PhD}$ from the Indian Institute of Technology (IIT), Kharagpur, India, in 2000 and 2006 respectively. His research interest includes image and video processing, medical image analysis, object tracking, multimedia information retrieval from web and frequent pattern mining. He was awarded an Indo-US Research Fellow Award in 2008 by the Indo-US Science and Technology Forum, India and young scientist award by DST Government of India in 2007.

Shaila S.G received her M-Tech in Computer Science and Engineering in 2005 from M.V.Jayaraman College of Engineering, Vishweshwarayya Technological University, Bangalore, India. Currently she is a research scholar in the Department of Computer Applications, National Institute of Technology, Tiruchirappalli, India. Her research interest is Multimedia Information retrieval from distributed environment.
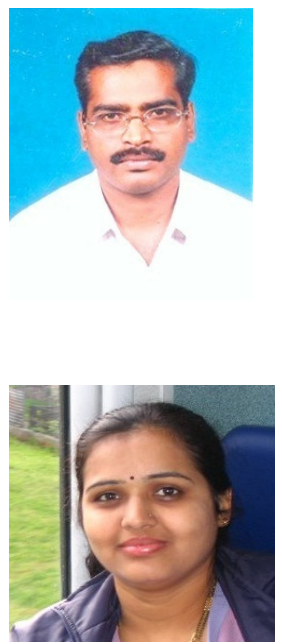